\title{
Suppression of Ecological Competition by Apex Predator
}

\author{
Taksu Cheor* and Shigemi Ohtd \\ * Laboratory of Physics, Kochi University of Technology, Tosa Yamada, Kochi 782-8502, Japan \\ $\dagger$ Institute of Particle and Nuclear Studies, KEK, Tsukuba, Ibaraki 305-0801, Japan
}

(Dated: August 11, 2003)

\begin{abstract}
In the framework of Lotka-Volterra dynamics with evolutionary parameter variation, it is shown that a system of two competing species which is evolutionarily unstable, if left to themselves, is stabilized by a commmon predator preying on both of them. Game-theoretic implications of the results are also discussed.
\end{abstract}

PACS numbers: 87.23.-n, 05.45.-a, 89.75.Da

Keywords: Population dynamics, Lotka-Volterra system, Game theory, Ecological stability

From the dominant plants in forest vegetation to the wild beasts in savanna, an often encountered ecological paradox exists in the form of stabilizing influence of the top predator. While two species in direct competition rarely form a stable ecosystem, they often coexist under the dominance of a common predator [1]. The most illustrative example is found in the trophic pyramids, where the apex predator, the most savage aggressor of all, seem to act as the key guarantor of the stability of the whole system 22]. For species with intellectual capacity, this fact might be explained as a result of conscious effort of enlightened self-interest. But the peacekeeping function of the apex predator is so prevalent throughout ecosystems, that the existence of a simple and universal dynamics should be suspected.

The purpose of this paper is to understand the structure and stability of ecosystems composed of competing species in the framework of evolutionary population dynamics [3, 4]. The tool we employ is the Lotka-Volterra equation with adiabatic parameter variation [5]. In this approach, the ecological dynamics is determined by the time variation of the variables representing the population of the species, while the adiabatic parameter variation represents the behavioral evolution of the species. The viability of a species in this framework is judged both by the short-time ecological stability of the orbit and also by the long-term evolutionary stability of the shifting parameters.

We focus specifically on a system that consists of two self-sustaining but competing species and an apex predator who preys over both competitors. We show that the system evolves towards an evolutionarily stable configuration in which the warring preys are tamed into the peaceful coexistence. This is in contrast to the case of two competitors left to themselves, in which there are no evolutionarily stable solution for coexistence, and "arms race" drives one of the competitors into eventual extinction. We also show that our results can be interpreted in a game-theoretic language as the apex predator turning

\footnotetext{
${ }^{*}$ E-mail taksu.cheon@kochi-tech.ac.jp
}

${ }^{\dagger}$ E-mail shigemi.ohta@kek.jp

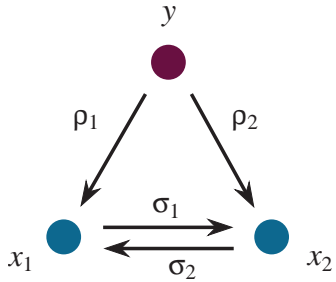

FIG. 1: A symbolic diagram showing the pecking order of the three species system described by (1). The arrows represent the aggression and predation with the specified intensities.

the prisoner's dilemma between two competitors into a collaborative game.

Suppose there are two populations of competing species $x_{1}$ and $x_{2}$ which are preyed upon by a common predator population $y$ (Fig. 11). We describe the ecological dynamics of this system by the standard LotkaVolterra equation

$$
\begin{aligned}
\dot{x}_{1} & =b_{1} x_{1}-a_{1} x_{1}^{2}-\sigma_{2} x_{1} x_{2}-\rho_{1} x_{1} y, \\
\dot{x}_{2} & =b_{2} x_{2}-a_{2} x_{2}^{2}-\sigma_{1} x_{1} x_{2}-\rho_{2} x_{2} y, \\
\dot{y} & =-d y+f \rho_{1} x_{1} y+f \rho_{2} x_{2} y .
\end{aligned}
$$

Here, $b_{1}, b_{2}$ are the reproduction rates for species $x_{1}, x_{2}$, and $a_{1}, a_{2}$, the environmental limitation factor to their growth. The coefficient $d$ is the decay rate for the predator $y$, and $f$, the efficiency of its predation. In the last equation, a term proportional to $y^{2}$ could be added for consistency with other equations, but this can be shown to introduce simply a technical complication without affecting the main line of our arguments. Also, specifying separate predation efficiencies for $x_{1}$ and $x_{2}$ makes no essential difference, because the result can be turned into the original form (1) with rescaling of variables. The parameters $\rho_{1}$ and $\rho_{2}$ are the aggression intensities of the apex predator $y$ towards $x_{1}$ and $x_{2}$, respectively. Similarly, $\sigma_{1}$ and $\sigma_{2}$ are the aggression intensities of $x_{1}$ to $x_{2}$ and of $x_{2}$ to $x_{1}$, respectively. We assume all parameters to be positive real numbers. At this stage, we treat all of them as fixed numbers, making no distinction between the Roman denominated "environmental" parameters and Greek denominated "behavioral" parameters. A 
nontrivial fixed point $x_{i}(t)=X_{i}, y(t)=Y\left(\dot{X}_{i}=\dot{Y}=0\right)$ with $i=1,2$ is given by

$$
\begin{aligned}
X_{1}= & \frac{1}{f} \cdot \frac{d\left(a_{2} \rho_{1}-\sigma_{2} \rho_{2}\right)-f\left(b_{2} \rho_{1}-b_{1} \rho_{2}\right) \rho_{2}}{a_{2} \rho_{1}^{2}+a_{1} \rho_{2}^{2}-\left(\sigma_{1}+\sigma_{2}\right) \rho_{1} \rho_{2}}, \\
X_{2}= & \frac{1}{f} \cdot \frac{d\left(a_{1} \rho_{2}-\sigma_{1} \rho_{1}\right)+f\left(b_{2} \rho_{1}-b_{1} \rho_{2}\right) \rho_{1}}{a_{2} \rho_{1}^{2}+a_{1} \rho_{2}^{2}-\left(\sigma_{1}+\sigma_{2}\right) \rho_{1} \rho_{2}}, \\
Y= & -\frac{d}{f} \cdot \frac{a_{1} a_{2}-\sigma_{1} \sigma_{2}}{a_{2} \rho_{1}^{2}+a_{1} \rho_{2}^{2}-\left(\sigma_{1}+\sigma_{2}\right) \rho_{1} \rho_{2}} \\
& +\frac{\left(a_{2} b_{1}-b_{2} \sigma_{2}\right) \rho_{1}+\left(a_{1} b_{2}-b_{1} \sigma_{1}\right) \rho_{2}}{a_{2} \rho_{1}^{2}+a_{1} \rho_{2}^{2}-\left(\sigma_{1}+\sigma_{2}\right) \rho_{1} \rho_{2}} .
\end{aligned}
$$

The stability of the fixed point is determined by the behavior of the linearized map

$$
M=\left(\begin{array}{ccc}
-a_{1} X_{1} & -\sigma_{2} X_{1} & -\rho_{1} X_{1} \\
-\sigma_{1} X_{2} & -a_{2} X_{2} & -\rho_{2} X_{2} \\
f \rho_{1} Y & f \rho_{2} Y & 0
\end{array}\right) .
$$

Namely, the fixed point is stable when real part of all the eigenvalues $\lambda$ of $M$ determined by

$$
|\lambda I-M|=0
$$

is negative.

When the fixed point is of stable, attracting sort, neighboring orbits form an absorbing spiral in phase space. We now assume that evolutionary pressure of selection and adaptation are at work. We can then regard aggression intensities $\rho_{1}, \rho_{2}, \sigma_{1}$ and $\sigma_{2}$ as evolutionarily adjustable parameters which evolve along the path that simultaneously increase the functions $X_{1}\left[\sigma_{1}\right], X_{2}\left[\sigma_{2}\right]$ and $Y\left[\rho_{1}, \rho_{2}\right]$ until they reach the optimal values. There are several indirect pieces of evidence supporting the existence of this type of adiabatic evolution among real-life ecosystems [6, 7]. It is convenient to start with the maximization condition for the apex predator $\partial Y /\left.\partial \rho_{1}\right|_{\rho_{1}^{\star}}=0$ and $\partial Y /\left.\partial \rho_{2}\right|_{\rho_{2}^{\star}}=0$. We then have the relations

$$
\begin{aligned}
& \rho_{1}^{\star}=\frac{d}{f} \cdot \frac{2 a_{1} a_{2} b_{1}-b_{1} \sigma_{+} \sigma_{1}+a_{1} b_{2} \sigma_{-}}{a_{2} b_{1}^{2}+a_{1} b_{2}^{2}-b_{1} b_{2} \sigma_{+}}, \\
& \rho_{2}^{\star}=\frac{d}{f} \cdot \frac{2 a_{1} a_{2} b_{2}-b_{2} \sigma_{+} \sigma_{2}-a_{2} b_{1} \sigma_{-}}{a_{2} b_{1}^{2}+a_{1} b_{2}^{2}-b_{1} b_{2} \sigma_{+}} .
\end{aligned}
$$

These conditions give the expressions

$$
\begin{aligned}
X_{1}^{\star} & =\frac{2 a_{2} b_{1}-b_{2} \sigma_{+}}{4 a_{1} a_{2}-\sigma_{+}^{2}}, \\
X_{2}^{\star} & =\frac{2 a_{1} b_{2}-b_{1} \sigma_{+}}{4 a_{1} a_{2}-\sigma_{+}^{2}}, \\
Y^{\star} & =\frac{f}{d} \cdot \frac{a_{2} b_{1}^{2}+a_{1} b_{2}^{2}-b_{1} b_{2} \sigma_{+}}{4 a_{1} a_{2}-\sigma_{+}^{2}} .
\end{aligned}
$$

The quantities $-X_{1}^{\star}$ and $-X_{2}^{\star}$ as functions of $\sigma_{1}$ and $\sigma_{2}$ act as the "potential surface" for the variation of $\sigma_{1}$ and $\sigma_{2}$. In (5) and (6), the notation $\sigma_{ \pm} \equiv \sigma_{1} \pm \sigma_{2}$ is used. With the definitions $\alpha \equiv \sqrt{a_{1} a_{2}}$ and $\beta \equiv \sqrt{a_{2} / a_{1}} \cdot b_{1} / b_{2}$,

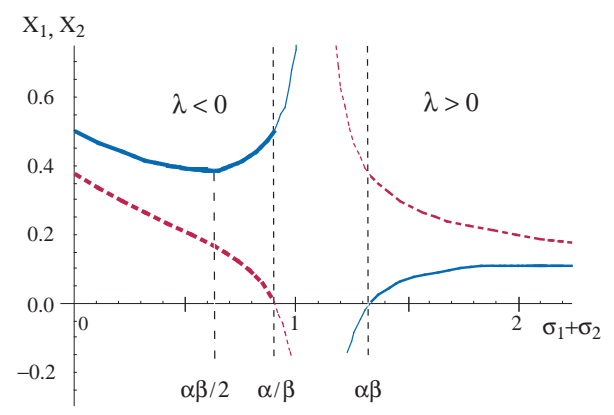

FIG. 2: Fixed point coordinates $X_{1}^{\star}$ and $X_{2}^{\star}$ as functions of $\sigma_{1}+\sigma_{2}$. The parameters are $a_{1}=1, b_{1}=1, a_{2}=1.2$, $b_{2}=0.9$ and $d=2$. Solid line represents $X_{1}^{\star}$ and the dahed $X_{2}^{\star}$. The fixed point is stable in the region below $\alpha / \beta$ but unstable above $\alpha \beta$. The region in between is unphysical.
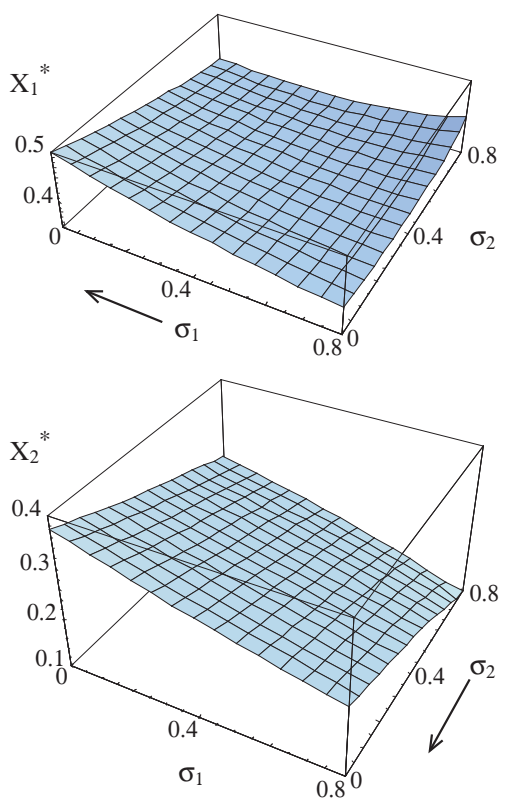

FIG. 3: Fixed point coordinates $X_{1}^{\star}$ and $X_{2}^{\star}$ as functions of $\sigma_{1}$ and $\sigma_{2}$. The parameters are $a_{1}=1, b_{1}=1, a_{2}=1.2$, $b_{2}=0.9$ and $d=2$. In the region $\sigma_{1}+\sigma_{2}<\sigma_{c r}=\alpha \beta / 2$ $=a_{2} b_{1} / b_{2}$, both $\sigma_{1}$ and $\sigma_{2}$ have to be decreased to make $X_{1}$ and $X_{2}$ larger.

valid parameter range for $X_{i}$ and $Y$ being positive and stable $(\Re \lambda<0)$ is given by

$$
\sigma_{1}+\sigma_{2}<\min \{\alpha \beta, \alpha / \beta\}
$$

That stability requirement is satisfied can be checked by the fact that all the coefficients of the third order polynomial equation (4) are of same sign within this parameter range.

The evolution of $\sigma_{1}$ and $\sigma_{2}$ depends on their starting 

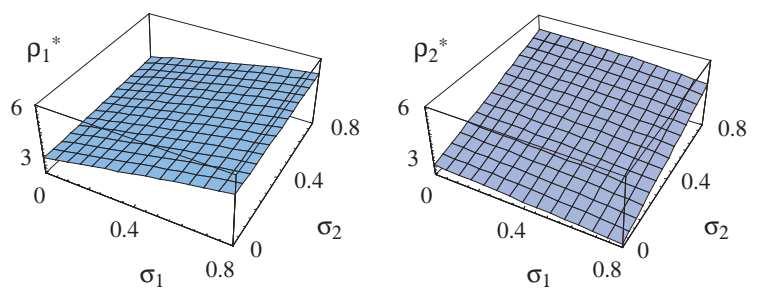

FIG. 4: Aggression intensities $\rho_{1}^{\star}$ and $\rho_{2}^{\star}$ as functions of $\sigma_{1}$ and $\sigma_{2}$. The parameters are $a_{1}=1, b_{1}=1, a_{2}=1.2, b_{2}=0.9$, $d=2$ and $f=0.7$.

values. With a straightforward calculation, we obtain

$$
\begin{array}{r}
\frac{\partial X_{1}^{\star}}{\partial \sigma_{1}}<0, \frac{\partial X_{2}^{\star}}{\partial \sigma_{2}}<0: \sigma_{1}+\sigma_{2}<\sigma_{c r}, \\
\frac{\partial X_{1}^{\star}}{\partial \sigma_{1}} \cdot \frac{\partial X_{2}^{\star}}{\partial \sigma_{2}}<0: \sigma_{1}+\sigma_{2}>\sigma_{c r},
\end{array}
$$

within the range of (7). The critical aggression intensity $\sigma_{c r}$ is given by

$$
\sigma_{c r} \equiv \max \{\alpha \beta / 2, \alpha / 2 \beta\} .
$$

If the sum $\sigma_{1}+\sigma_{2}$ is bellow $\sigma_{c r}$, both $\sigma_{1}$ and $\sigma_{2}$ will move toward $\sigma_{1}=\sigma_{2}=0$. Namely, two competing species shall settle for a peaceful coexistence as the common preys of a predator $y$. On the other hand, if the sum starts above critical value, $\sigma_{1}$ and $\sigma_{2}$ will increase until one of the competing species is extinct at that critical value. The situation becomes immediately clear with a glance at numerical example depicted in Fig. 2 and Fig. 3

A crucial point is that the master $y$ acts as a punisher, according to (5), that inhibits the increase of $\sigma_{1}$ and $\sigma_{2}$. Fig. [4 serves as a graphical illustration of this effect; Increasing $\sigma_{i}$ will induce an increase of $\rho_{i}$ that incur the damage upon $x_{i}$. We stress that no special mechanism is assumed for $y$ to police the system in the outset, yet the dynamics seems to explain our common sense observation about apex predators.

An intriguing fact is that the critical value $\sigma_{c r}$ is inversely proportional to the natural population of one of the prey species, $b_{1} / a_{1}$ or $b_{2} / a_{2}$. This means that the coexistence of competing species under common predator becomes a less likelier outcome for a system with richer resources. This seems to give a partial explanation to the experimentally observed decrease of species at the base levels of trophic pyramids [6].

We next consider the case where the predator leaves the scene, namely $y=0$ (Fig. [5). By setting $\rho_{1}=\rho_{2}$ $=0$, we obtain, in place of $(6)$,

$$
\begin{aligned}
& X_{1}^{\star}=\frac{a_{2} b_{1}-\sigma_{2} b_{2}}{a_{1} a_{2}-\sigma_{1} \sigma_{2}} \\
& X_{2}^{\star}=\frac{a_{1} b_{2}-\sigma_{1} b_{1}}{a_{1} a_{2}-\sigma_{1} \sigma_{2}} .
\end{aligned}
$$

The linearized map now takes a two-by-two matrix form

$$
M=\left(\begin{array}{ll}
-a_{1} X_{1} & -\sigma_{2} X_{1} \\
-\sigma_{1} X_{2} & -a_{2} X_{2}
\end{array}\right)
$$

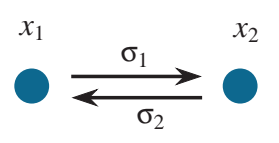

FIG. 5: A symbolic diagram showing the two competing species described by (1) with $\rho_{1}=\rho_{2}=0$.
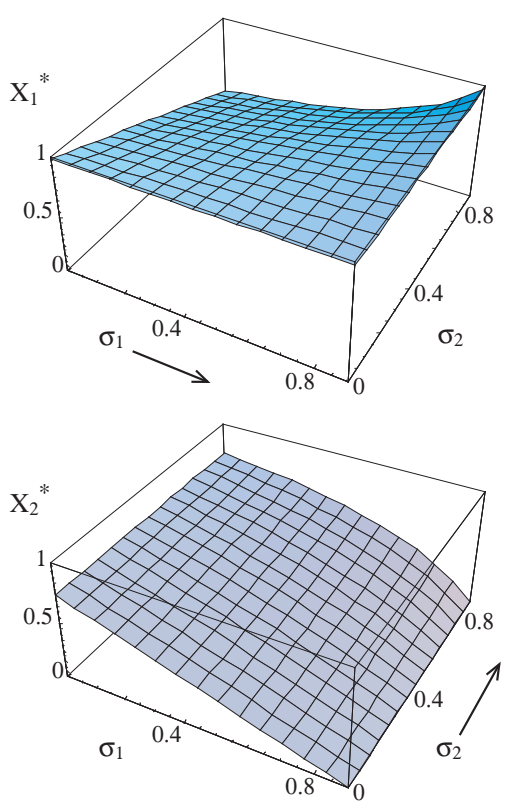

FIG. 6: Fixed point coordinates $X_{1}^{\star}$ and $X_{2}^{\star}$ for the case without the apex predator as functions of $\sigma_{1}$ and $\sigma_{2} . X_{1}^{\star}$ will increase $\sigma_{1}$ and $X_{2}^{\star}$ will increase $\sigma_{2}$ to achieve local advantage, which will eventually doom $X_{2}^{\star}$ to extinction. The parameters are $a_{1}=1, b_{1}=1, a_{2}=1.2$, and $b_{2}=0.9$.

in place of (3). The straightforward calculation gives the condition for $X_{1}^{\star}$ and $X_{2}^{\star}$ to be a viable fixed point, namely, $X_{1}^{\star}, X_{2}^{\star}>0, \Re \lambda<0$, in terms of the allowed region for the aggression intensity as

$$
\sigma_{1}<\min \{\alpha, \alpha \beta\}, \quad \sigma_{2}<\min \{\alpha, \alpha / \beta\} .
$$

However, within this region, we can easily check the relation

$$
\frac{\partial X_{1}^{\star}}{\partial \sigma_{1}}>0, \frac{\partial X_{2}^{\star}}{\partial \sigma_{2}}>0 .
$$

Therefore, in this case, both $\sigma_{1}$ or $\sigma_{2}$ shall eventually be increased beyond the range (12), and there is no evolutionarily stable coexisting solutions for two competing species. Namely, in the absence of the common master, one of the competing species is always driven to extinction by arms race of increasing $\sigma_{1}$ and $\sigma_{2}$. An example of this case is illustrated in Fig. [6

In order to fully understand the generic shape of the ecosystem, we would have to generalize our arguments to more trophic levels than two, and also more species than two within a single trophic level. To this end, this 
TABLE I: The game table $X_{1}^{\star}\left[\sigma_{1}, \sigma_{2}\right]$ for $x_{1}$ discretized at $\sigma_{1}, \sigma_{2}=a / 3$ (low aggressiveness, "dove") and $2 a / 3$ (high aggressiveness, "hawk"). Left hand side is the table for the case with apex predator (6), and the right hand side, without apex predator (10). The game table $X_{2}^{\star}\left[\sigma_{1}, \sigma_{2}\right]$ for $x_{2}$ is obtained by transposition of raw and column. The Nash equilibrium is indicated with boldface.
With Apex Predator

\begin{tabular}{c|cc}
$\sigma_{1} \backslash^{\sigma_{2}}$ & $\begin{array}{l}a / 3 \\
\text { dove }\end{array}$ & $\begin{array}{l}2 a / 3 \\
\text { hawk }\end{array}$ \\
\hline $\begin{array}{l}a / 3 \\
\text { dove }\end{array}$ & $\frac{\mathbf{3 b}}{\mathbf{8 a}}$ & $\frac{3 b}{9 a}$ \\
$\begin{array}{l}2 a / 3 \\
\text { hawk }\end{array}$ & $\frac{3 b}{9 a}$ & $\frac{3 b}{10 a}$
\end{tabular}

No Apex Predator

\begin{tabular}{c|cc}
$\sigma_{1} \backslash{ }^{\sigma_{2}}$ & $\begin{array}{l}a / 3 \\
\text { dove }\end{array}$ & $\begin{array}{l}2 a / 3 \\
\text { hawk }\end{array}$ \\
\hline $\begin{array}{l}a / 3 \\
\text { dove }\end{array}$ & $\frac{6 b}{8 a}$ & $\frac{6 b}{14 a}$ \\
$2 a / 3$ & $\frac{6 b}{7 a}$ & $\frac{\mathbf{6 b}}{\mathbf{1 0 a}}$
\end{tabular}

work, in combination to our previous work, is but a modest start. More involved and sophisticated approaches of both numerical and analytical nature may have to be called for $[6,8,9]$. In the current work, no precise specification for the evolutionary dynamics of behavioral parameters has been required. While we stress that this is an advantage, construction and analysis of more detailed models with such specifications are attractive possibilities.

Finally, some remarks in the broader context of game theory [10] are in order. There is an obvious gametheoretic interpretation of the results (6) and (10). For the sake of simplicity, let us set $a_{1}=a_{2}=a$ and $b_{1}=b_{2}=b$. We now regard $\sigma_{i}(i=1,2)$ as the control parameter of the strategy of population $x_{i}$ for the game played between $x_{1}$ and $x_{2}$ whose payoff tables are given by $X_{1}^{\star}\left[\sigma_{1}, \sigma_{2}\right]$ and $X_{2}^{\star}\left[\sigma_{1}, \sigma_{2}\right]$. To facilitate the understanding, the game tables for two discretized points for $\sigma_{1}$ and $\sigma_{2}$ are tabulated in Table I. For the case without the common predator, $(10)$, the game is a continuous strategy version of prisoner's dilemma [11, 12], whose outcome is $\sigma_{1} \rightarrow a, \sigma_{2} \rightarrow a$ which leads to the extinction of either $x_{1}$ or $x_{2}$. With the introduction of the apex predator, the game table is turned into one of collaborative game, whose outcome is the coexistence $\sigma_{1} \rightarrow 0$ and $\sigma_{2} \rightarrow 0$. Note that the game table is symmetrized under the presence of the apex predator; $X_{2}^{\star}\left[\sigma_{1}, \sigma_{2}\right]=$ $X_{1}^{\star}\left[\sigma_{2}, \sigma_{1}\right]$. This could be interpreted as the sign of altruistic behavior [13]. Advantage of having the apex predator as a "law enforcer" is evident, and the loss of half of the populations to the predation would be an acceptable tradeoff.

In summary, we have established, for Lotka-Volterra systems with evolutionary parameter variation, that two competing species are evolutionarily unstable, but can be stabilized by the introduction of an apex predator. We hope this to be a start for systematic understanding of stable ecosystems.

The authors wish to express gratitudes to Professors Kazuo Takayanagi, Toshiya Kawai and David Greene for helpful discussions and useful comments.
[1] L.A. Dugatkin (ed.), Model Systems in Behavioral Ecology: Integrating conceptual, theoretical, and empirical approaches, (Princeton Univ. Press, Princeton, 2001).

[2] C. Elton, Animal Ecology, (Sidgewick and Jackson, London, 1927).

[3] R.M. May, Stability and Complexity in Model Ecosystems, Priceton Univ. Press, Princeton, 1974).

[4] J. Hofbauer and K. Sigmund:, The Theory of Evolution and Dynamical Systems, (Cambridge Univ. Press, Cambridge, 1988).

[5] T. Cheon, Evolutionary stability of ecological hierarchy, Phys. Rev. Lett. 90 (2003) 258105(4).

[6] M. Lässig, U. Batolla, S.C. Manrubia, A. Valleriani Shape of ecological networks, Phys. Rev. Lett. 86 (2001) 44184421.

[7] S. Foitzik, C.J. DeHeer, D.N. Hunjan and J.M. Herbers, Coevolution in host-parasite systems: Behavioural strate- gies of slave-making ants and their hosts, Proc. Roy. Soc. London B268 (2001) 1139-1146.

[8] V.M. de Oliviera and J.F. Fontanari, Complimentarity and diversity in a soluble model ecosystem, Phys. Rev. Lett. 89 (2002) 148101(4).

[9] D. Chowdhury, D. Stauffer and A. Kunwar, Unification of small and large time scales for biological evolution, Phys. Rev. Lett. 90 (2003) 068101(4).

[10] J. Maynard Smith, Evolution and the Theory of Games, (Cambridge Univ. Press, Cambridge, 1982).

[11] R. Axelrod, The Evolution of Cooperation, (Basic Books, New York, 1984).

[12] H. Gintis, Game Theory Evolving, (Princeton Univ. Press, Princeton, 2000).

[13] T. Cheon, Altruistic duality in evolutionary game theory, preprint arXiv.org cond-mat/0305351 (2003). 